\title{
Tratamento cirúrgico da lesão do óstio da coronária esquerda
}

Luiz Carlos Bento de SOUZA*, Paulo CHACCUR*. Jarbas J. DINKHUYSEN*, Savério ANGRISANI NETO**, Antoninho S. ARNONI***, Camilo ABDULMASSIH NETO*, Haroldo B. BARROSO**. Ricardo PAVANELLO**. Leopoldo S. PIEGAS ${ }^{\star}$, Paulo P. PAULISTA ${ }^{\star \star \star}$, J. Eduardo M. R. SOUSA ${ }^{\star}$, Adib D. JATENE ${ }^{\star \star}$

RBCCV 44205-65

SOUZA, L. C. B.; CHACCUR, P.; DINKHUYSEN, J. J.; ANGRISANINETO, S.; ARNONI, A. S.; ABDULMASSIH NETO, C.; BARROSO, H. B.; PAVANELlO, R.; PIEGAS, L. S.; PAUlista, P. P.; SOUSA, J. E. M. R.; JATENE, A. D. - Tratamento cirúrgico da lesão do óstio da coronária esquerda. Rev. Bras. Cir. Cardiovasc., 3(3): 182-188, 1988.

RESUMO: A lesāo do óstio da coronária esquerda pode ser tratada cirurgicamente de duas maneiras: revascularização dos principais ramos arteriais da coronária esquerda (DA, diagonais, circunflexa), ou ampliando a área estenosada, portanto, realizando um ataque direto à lesão. O presente trabalho apresenta nossa experiência com 4 pacientes portadores de lesão do óstio da coronária esquerda e que foram tratados por ampliação da regiāo lesada. Todos os pacientes eram do sexo masculino, com idades de 56,59 , 61 e 68 anos. Em 3 deles, havia presença de fatores de risco de doença coronária (hipertensâo, diabetes, tabagismo) e, em 1, antecedente luético. Dois pacientes apresentavam quadro de angina instável, 1 apresentava angina estável e outro era assintomático, porém com teste ergométrico positivo. Do ponto de vista cinecoronariográfico, todos apresentavam lesāo importante $(>70 \%)$ do óstio da coronária esquerda, sem lesōes obstrutivas nas porçōes distais. A cirurgia foi conduzida com uso de circulação extracorpórea, hipotermia moderada e solução cardioplégica. Realizou-se aortotomia transversal direcionada posteriormente ao seio de Valsalva coronariano esquerdo, seccionando-se o óstio CE estenosado e prolongando-se até mais ou menos $1 \mathrm{~cm}$ pelo tronco da CE. A ampliação foi levada a efeito com segmento de veia safena em 3 casos e, em outro, retalho de pericárdio bovino tratado. $O$ tempo de perfusão variou de 45 a 85 minutos (média de 64 minutos) e o tempo de clampeamento foi de 34 a 62 minutos (média de 45 minutos). Nāo ocorreram complicaçōes dignas de nota no pós-operatório imediato, nāo havendo mortalidade hospitalar. Os pacientes evoluem em um período de 3 meses a 1 ano e 8 meses. Todos estão assintomáticos, com vida normal. Os autores concluem que a ampliação do óstio da CE é uma boa opção técnica a ser utilizada em lesōes deste tipo, que não se acompanhem de obstruçōes distais nos demais ramos arteriais.

DESCRITORES: óstio da coronária, cirurgia.

\section{INTRODUÇÃO}

A estenose de 1 ou dos 2 óstios coronários é uma eventualidade que pode ocorrer em uma série de doenças, tais como: aortite sifilitica ${ }^{12}$, doença de Takayasu ${ }^{1 .}$ ${ }^{6}$, estenose congênita ${ }^{8}$, estenose aórtica supravalvar ${ }^{9}$, após substituição da valva aórtica ${ }^{10}$ e doença ateromatosa $a^{5}$.

Embora a incidência da lesão isolada do óstio coronário seja uma entidade pouco freqüente - na experiência de HUTTER et alii ${ }^{7}$ representa $0,093 \%$ de todos os pa-

\footnotetext{
Trabalho realizado no Hospital do Coração a Associaçăo do Sanatório Sírio e no Instituto Dante Pazzanese de Cardiologia. Sảo Paulo, SP, Brasil. Apresentado ao 15 : Congresso Nacional de Cirurgia Cardiaca. Rio de Janeiro, RJ, 7 e 8 de abril, 1988.

- Do Hospital do Coração e do Instituto Dante Pazzanese de Cardiologia.

*. Do Hospital do Coração.

... Do Instituto Dante Pazzanese de Cardiologia.

Endereço para separatas: Luiz Carlos Bento de Souza. Rua Desembargador Eliseu Guilherme, 123. 04004 Paraiso. São Paulo, SP, Brasil.
} 
SOUZA, L. C. B.; CHACCUR, P.; DINKHUYSEN, J. J.; ANGRISANI NETO, S.; ARNONI, A. S.; ABDULMASSIH NETO, C.; BARROSO. H. B.; PAVANELLO, R.; PIEGAS, L. S.; PAULISTA, P. P.; SOUSA, J. E. M. R.; JATENE, A. D. - Tratamento cirúrgico da lesão do óstio da coronária esquerda. Rev. Bras. Cir. Cardiovasc., 3(3): 182-188, 1988.

cientes submetidos a cinecoronariografia; PRITCHARD et alii" encontraram 4 casos em 3000 cinecoronariografias $(0,13 \%)$, COHEN et alii ${ }^{2}, 3$ casos, em 1200 estudos $(0,25 \%)$ - representa um risco igual ao das lesōes do tronco da coronária esquerda.

A finalidade do presente trabalho é a de estudar a opção do tratamento cirúrgico dessa lesão, com ataque direto, realizando a ampliação da área estenosada, ao invés de proceder aos métodos havituais de revascularização miocárdica usando as pontes de safena, ou anastomose mamária-coronária.

\section{MATERIAL E MÉTODOS}

No período entre janeiro de 1986 a dezembro de 1987, foram operados, em um dos hospitais de nosso Serviço, 1160 pacientes submetidos a revascularização do miocárdio, sendo que, em 4 deles $(0,34 \%)$, foi realizada ampliação da lesão ostial.

Todos os pacientes eram do sexo masculino, com idades de 56, 59, 61 e 68 anos. Em 3 casos, julgou-se a lesão ser devida a aterosclerose, com presença de fatores de risco, tais como: hipertensão arterial, diabetes e tabagismo. Em 1 caso, a lesão foi devida a aortite sifilítica cicatrizada.

Todos os pacientes negaram-se a fazer reestudo hemodinâmico para avaliação anatômica da correção.

No que diz respeito aos sintomas, 2 pacientes apresentavam quadro de angina instável, 1 angina estável e outro era assintomático, porém com teste ergométrico positivo.

O estudo cinecoronariográfico mostrou lesão superior a $70 \%$ em todos os casos. Apenas 1 paciente apresentava lesão de $50 \%$ no terço inicial da descendente anterior, que foi considerada de pequena importância. A funçāo ventricular esquerda estava preservada em todos os pacientes, com frações de ejeção $\geqslant 0,50$.

A cirurgia foi conduzida com o auxílio de circulação extracorpórea, hipotermia moderada e solução cardioplégica cristalóide como método de proteção miocárdica.

Realizou-se uma aortotomia transversal prolongada, posteriormente, pela direita, ao seio da Valsalva esquerdo, em direção ao óstio coronário estenosado (Figura 1), seccionando-o e estendendo a incisão por mais $10 \mathrm{~mm}$ pelo tronco da coronária esquerda (Figura 2).

A ampliação foi levada a efeito com retalho de veia safena, em 3 casos, e de pericárdio bovino tratado, em 1.

Iniciou-se a sutura com colocação de ponto em U, de monofilamento 5-0, na extremidade do enxerto e pas-

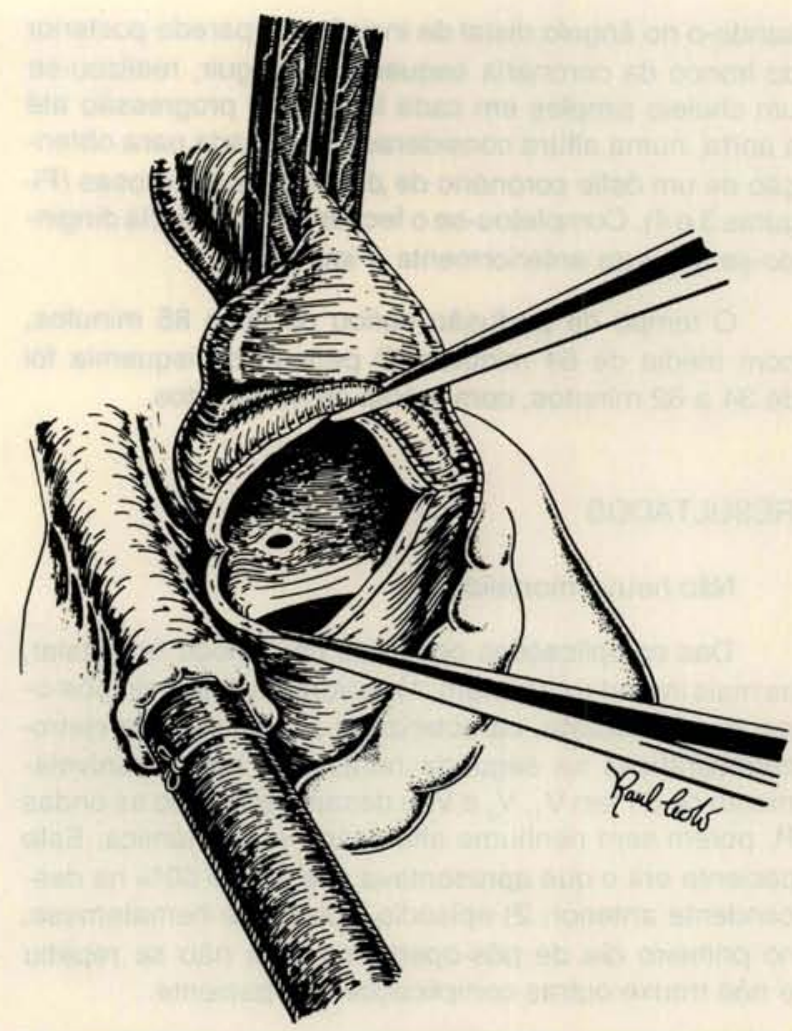

Fig. 1 - Aortotomia transversal dirigida para o óstio coronário esquerdo.

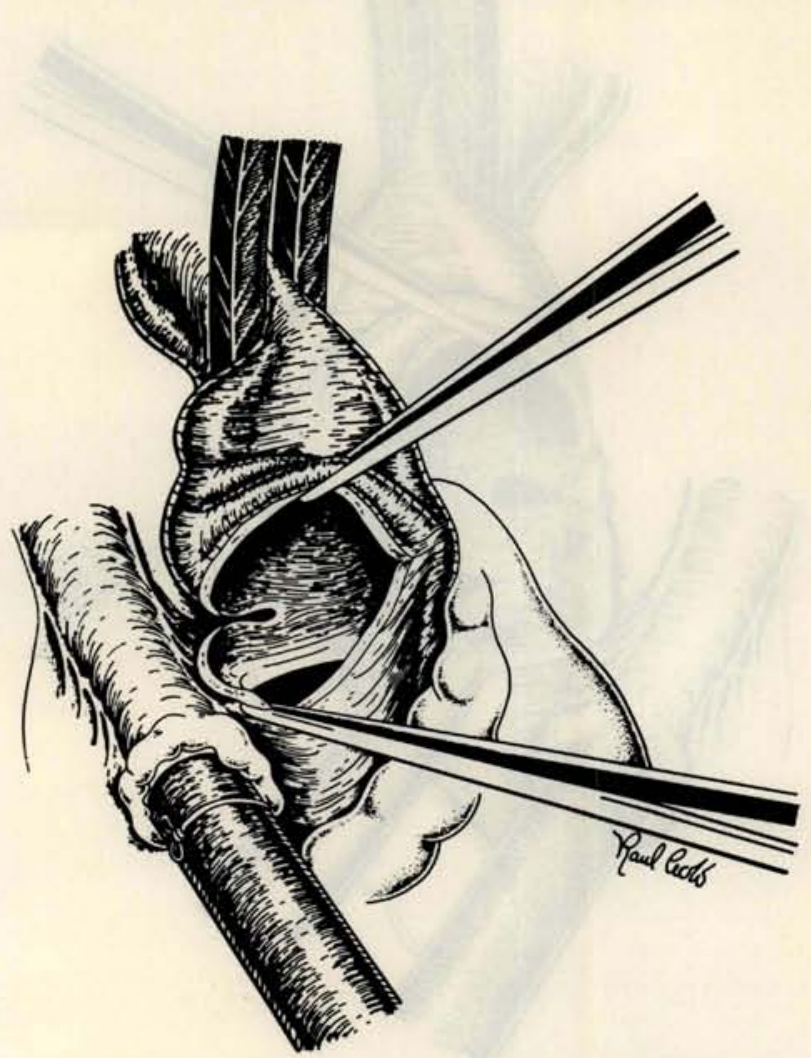

Fig. 2-Estensăo da incisão, pelo óstio e parede posterior do tronco da coronária esquerda. 
sando-o no ângulo distal da incisão, na parede posterior do tronco da coronária esquerda. A seguir, realizou-se um chuleio simples em cada lado, com progressão até a aorta, numa altura considerada adequada para obtenção de um óstio coronário de dimensōes generosas ( $\mathrm{Fi}$ guras 3 e 4). Completou-se o fechamento da aorta dirigindo-se a sutura anteriormente (Figura 5 ).

O tempo de perfusão variou de 45 a 85 minutos, com média de 64 minutos. $O$ período de isquemia foi de 34 a 62 minutos, com média de 45 minutos.

\section{RESULTADOS}

Não houve mortalidade.

Das complicações ocorridas no periodo hospitalar, as mais importantes foram: 1) quadro de infarto no pós-operatório imediato, caracterizado por alteraçōes eletrocardiográficas na segunda hora, com supradesnivelamento de ST em $V_{1}, V_{2}$ e $V_{3}$ e desaparecimento as ondas $\mathrm{R}$, porém sem nenhuma alteração hemodinâmica. Este paciente era o que apresentava a lesão de $50 \%$ na descendente anterior; 2) episódio isolado de hematemese, no primeiro dia de pós-operatório, que não se repetiu e não trouxe outras complicaçōes ao paciente.

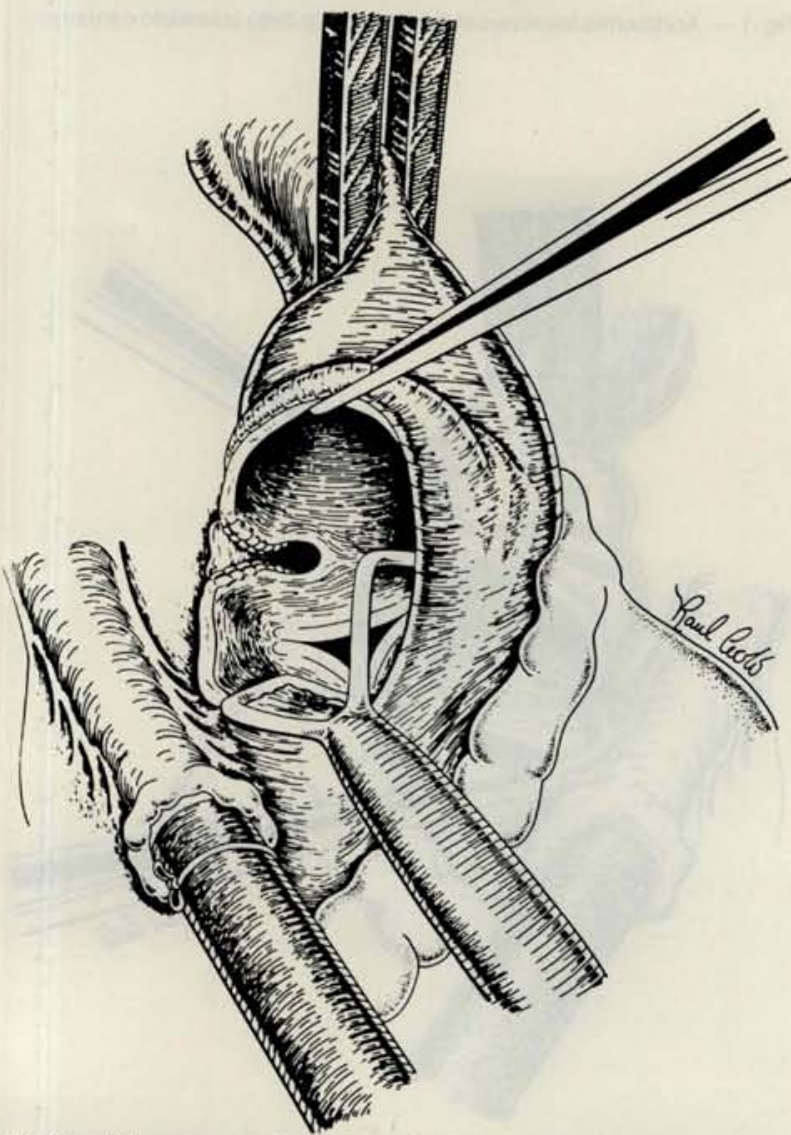

Fig. 3-Sutura do enxerto nas paredes laterais do tronco da coronária esquerda e aorta.

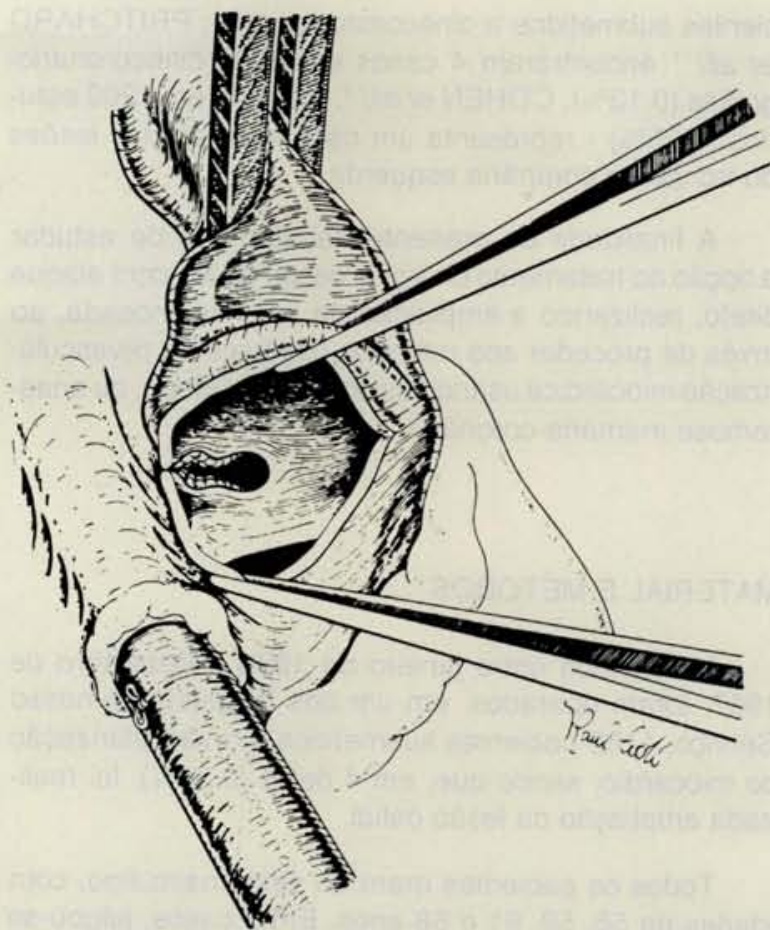

Fig. 4-Aproximaçâo das bordas da aorta configurando novo ośtio de dimensōes normais.

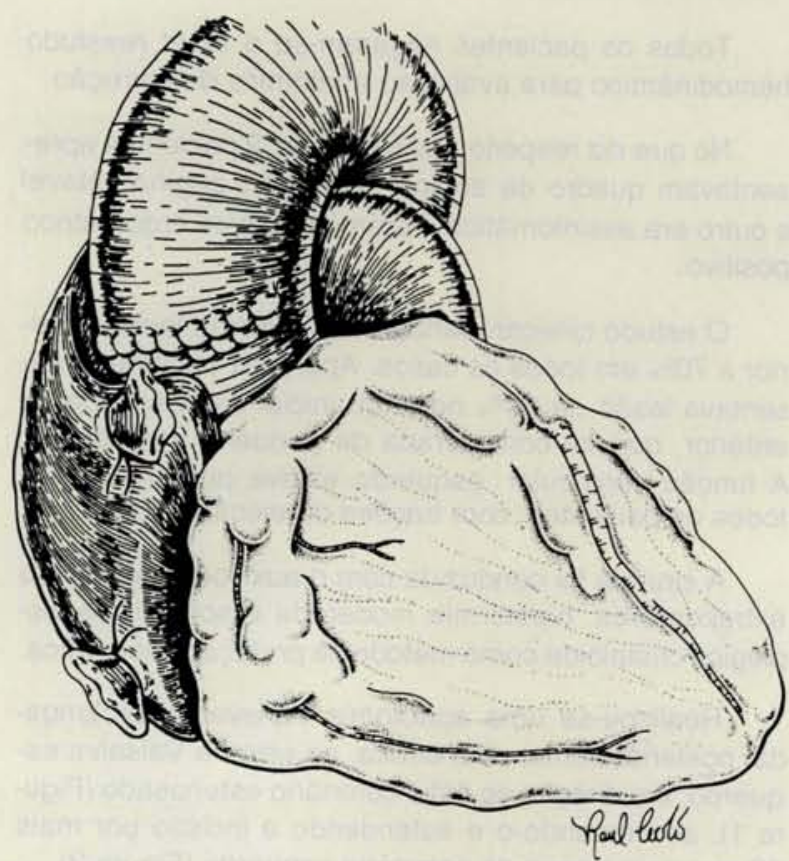

Fig. 5- Aortorrafia completada

Todos os pacientes tiveram alta hospitalar em boas condições clínicas, em média no 10 : dia de pós-operatório $\left(8^{\circ}, 9^{\circ}, 11\right.$ : e 12:). 

lesão do óstio da coronária esquerda. Rev. Bras. Cir. Cardiovasc., 3(3): 182-188, 1988.

O primeiro paciente, operado em novembro de 1986 , agora com 16 meses de evolução, apresenta-se assintomático, desenvolvendo plenamente sua atividade profissional (Medicina). O ecocardiograma recente mostrou função ventricular normal com fração de ejeção de 0,80 , sem outras anormalidades. $O$ teste de esforço mostrou significativa melhora em relação ao pré-operatório.

O segundo paciente, submetido a cirurgia em junho de 1987, tem, agora, 10 meses de evolução. Está assintomático, em vida normal. Fez teste ergométrico com radioisótopo (Thalium), que foi negativo para isquemia miocárdiça.

O terceiro paciente, operado em julho de 1987, evolui, agora com 9 meses de pós-operatório, estando assintomático, com vida normal e trabalhando. Tem teste ergométrico negativo.

O quarto paciente submeteu-se a intervenção em novembro de 1987. Aos 4 meses de evolução, mostra-se assintomático, tendo voltado à sua profissāo (motorista de veículos pesados). Não fez teste ergométrico de controle.

\section{COMENTÁRIOS}

$\mathrm{O}$ ataque direto às lesōes do óstio coronário fazem parte da história do tratamento cirúrgico da insuficiência coronária, tendo sido DUBOST et alii ${ }^{4}$, em 1960, quem, pela primeira vez, realizou, com auxílio da circulação extracorpórea, a desobstrução dos óstios coronários em caso de aortite sifilítica. Em 1964, CONNOLLY et alii ${ }^{3}$, advogaram, também, o emprego da desobstruçāo direta dos óstios coronários.

Mais recentemente, LEA et alii ${ }^{8}$ e MARTIN et alii ${ }^{9}$, em 1986 e 1988, respectivamente, demonstraram o uso prático da plastia do óstio coronário em casos considerados de etiologia congênita.

HUTTER et alii ${ }^{7}$, em 1985, em trabalho sobre incidência e tratamento da estenose do óstio coronário, concluem pela plena indicação das pontes aorto-coronárias com emprego de veia safena como o mais adequado procedimento, nessa situação.

A evidência do alto índice de obstruções nas pontes de safena, observada nos estudos de evolução tardia, evidentemente coloca em risco o resultado da cirurgia, predispondo a um significativo número de reintervençôes, principalmente nos pacientes jovens.

Achamos que o segmento de veia, colocado na posição de ampliação do óstio coronário, praticamente não terá chance de provocar reestenose, por duas causas fundamentais: em primeiro lugar, o enxerto não é tubular e sim laminar, e, em segundo lugar, a experiência tem demonstrado que as porçōes da veia nas proximidades da anastomose aórtica, em geral, são preservadas de lesões ateroscleróticas.

A impressão que fica é a de que o resultado tardio destas ampliaçōes deverá girar em função, principalmente, do aparecimento, ou não, de lesões nos ramos arteriais coronários.

Em função disto, achamos perfeitamente válido projetar-se este tipo de cirurgia para os casos de lesão de tronco da coronária esquerda, sem acometimento dos ramos, como já fora anteriormente preconizado, em 1968, por ZERBINI et alii ${ }^{13}$.

Os autores concluem ser este um procedimento plenamente válido para o tratamento cirúrgico da estenose dos óstios coronários; chamam a atenção para a possivel aplicação da técnica de ampliação, com retalho de veia, das lesōes do tronco da coronária esquerda, exposto após transecção do tronco pulmonar. 
SOUZA, L. C. B.; CHACCUR, P.; DINKHUYSEN, J. J.; ANGRISANI NETO, S.; ARNONI, A. S.; ABDULMASSIH NETO, C.; BARROSO, H. B.; PAVANELLO, R.; PIEGAS, L. S.; PAULISTA, P. P.; SOUSA, J. E. M. R.; JATENE, A. D. - Tratamento cirúrgico da lesão do óstio da coronária esquerda. Rev. Bras. Cir. Cardiovasc., 3(3): 182-188, 1988.

RBCCV 44205-65

SOUZA, L. C. B.; CHACCUR, P.; DINKHUYSEN, J. J.; ANGRISANINETO, S.; ARNONI, A. S.; ABDULMASSIH NETO, C.; BARROSO, H. B.; PAVANELLO, R.; PIEGAS, L. S.; PAULISTA, P. P.; SOUSA, J. E. M. R.; JATENE, A. D. - Surgical management of the left coronary ostial stenosis. Rev. Bras. Cir. Cardiovasc., 3(3): 182-188, 1988.

ABSTRACT: The surgical management of the left coronary mitral stenosis can be achieved by revascularizing individually its main branches (LAD, Cfx), or duing a direct approach over the stenosed area. The purpose of this paper is to present the results of the surgical enlargement of this lesion in 4 patients. All cases were males, aging 56, 59, 61 and 68 years old. Three cases had risk factors for coronary disease (hypertension, diabetes, heavy smokers), and 1 had syphilis. Two patients were in unstable angina, 1 presented stable angina, and the last one was assymptomatic, with a positive ergometric test. All patients showed obstructions more than $70 \%$ at the cinecoronariography, with no significant peripheral lesions. The operations were performed with the use of moderate hypothermia and crystaloid cardioplegic solution. A transverse aortotomy was made, extending posteriorly to the left coronary sinus, dividing the ostium, and entering $1 \mathrm{~cm}$ through the left main artery. In 3 cases, a saphenous vein patch was used for the enlargement. and bovine pericardium on the other one. The immediate postopertive period was uneventful, asn all patients were discharged from the hospital. The late follow-up at 20 months shows assymptomatic patients with normal life. The authors emphasize that the surgical widening of the coronary ostium seems to be a good technical option to treat coronary ostial stenosis, with no peripheral lesions.

DESCRIPTORS: coronary ostial stenosis, surgery.

\section{REFERÊNCIAS BIBLIOGRÁFICAS}

1 CIPRIANO, P. R.; SILVERMAN, J. F.; PERLROTH, M. G.; GRIEPP, R. B.; WEXLER, L. - Coronary arterial narrowing in Takayasu's aortitis. Am. J. Cardiol, 39(5): 744-750, 1977

2. COHEN, M. V.; COHN, P. F.; HEMAN, M. V.; GORLIN R. - Diagnosis and prognosis of main left coronary artery obstruction. Circulation, 45(Supl. 1): 57-65, 1972.

3. CONNOLLY, J. E.; ELDRIDGE, F. L.; CALVIN, J. W.; STEMMER, E. A. - Proximal coronary artery obstruction: Its aethiology and treatment by transaortic endarterectomy. $\quad$ N. Eng. J. Med. 271(5): 213-219, 1964.

4. DUBOST, C.; BLONDEAU, P.; PIWNICA, A.; WEISS, M.; LENFANT, C.; PASSELECQ, J.; GUERY, J. Syphilitic coronary obstruction: corrections under artificial heart-lung and profound hypothermia at $10: \mathrm{C}$. Surgery, 48(3): 540-547, 1960.

5. ENOS, W. F.; BEYER, J. C.; HOLMES, R. H. - Arteriosclerosis of the aortic sinuses. Am J. Clin. Pathol. 39(5): 506-511, 1963

6. FROVIG, A. G. \& LOKEN, A. C. - Syndrome of obliteration of the arterial branches of the aortic arch due to arteritis. Acta Psychiatr. Neurol. Scand. 26: 313-337, 1951.

7. HUTTER, J. A.; PASAOGLU, I.; WILLIAMS, B. T. - The incidence and management of coronary ostial stenosis. J. Cardiovasc. Surg.,26(6): 581-584, 1985.

8. LEA VI, J. W.; PAGE, D.; HAMMON, J. W. - Congenital ostial stenosis of the right coronary artery repaired by vein patch angioplasty. J. Thorac. Cardiovasc. Surg. 92(4): $796-798,1986$

9. MARTIN, M. M.; LEMMER Jr., J. H.; SHAFFER, E.; DICK II, M.: BOVE, E. L. - Obstruction to left coronary artery blood flow secondary to obliteration of the coronary ostium in supravalvular aortic stenosis. Ann. Thorac. Surg., 45(1): 16-20, 1988.

10. MIDDELL, A. I.; DeBOER, A.; BERMUdEZ, G. - Post perfusion coronary ostial stenosis. J. Thorac. Cardiovasc. Surg., 71(1): 72-80, 1976

11. PRITCHARD, C. L.; MUDD, J. G.; BARNER, H. B. Coronary ostial stenosis. Circulation., 52: 46-48, 1975.

12. SCHARFMAN, W. B.; WALLACH, J. B.; ANGRIST, A. Myocardial infarction due to syphilitic coronary ostial stenosis. Am. Heart. J., 40(4): 603-613, 1950.

13. ZERBINI, E. J.; SOUSA, J. E. M. R.; JATENE, A. D.; BITTENCOURT, D.; PILLEGI, F.; CAMPOS FILHO, C. M. - Tratamento cirúrgico da insuficiência coronária. Arq. Bras. Cardiol., 21(supl. 1): 33-40, 1968.

AGRADECIMENTO: Os autores agradecem a assistência do Sr. RAUL CECÍLIO MENEZES JÚNIOR, desenhista técnico, na ilustração esquemática dos tempos cirúrgicos. 
SOUZA, L. C. B.; CHACCUR, P.; DINKHUYSEN, J. J.; ANGRISANI NETO, S.; ARNONI, A. S.; ABDULMASSIH NETO, C.; BARROSO, H. B.; PAVANELlO, R.; PIEGAS, L. S.; PAULISTA, P. P.; SOUSA, J. E. M. R.; JATENE, A. D. - Tratamento cirúrgico da lesẫo do óstio da coronária esquerda. Rev. Bras. Cir. Cardiovasc., 3(3): 182-188, 1988.

\section{Discussão}

\section{DR. CARLOS FIGUEIROA}

\section{Belo Horizonte, $M G$}

Inicialmente, gostaria de agradecer o convite da comissão organizadora, para discutir o trabalho do Dr. Luiz Carlos. As lesões do óstio da coronária, como bem salientou o nosso prezado Luiz, é uma condição realmente rara; elas se apresentam com maior frequeência na lesão sifilitica, na aterosclerose, na doença congênita e em algumas situaçōes de retroca da valva aórtica, em que podemos ter obstrução dos óstios da coronária e iatrogênica, por uma colocação da prótese bem acima do anel da valva aórtica; (slide) no curto tempo que tivemos para revisar a literatura, encontrei, recentemente, os últimos trabalhos, já referidos pelo $\mathrm{Dr}$. Luiz e esta é a cirurgia proposta pelo grupo de Tenessee, sobre lesões de óstio da coronária direita, que faz a plastia com fragmentos de veia; o outro trabalho, também já referido aqui, que mostra o grupo de Michigan, sobre o emprego de pericárdio autógeno no óstio da coronária esquerda, nas lesões congênitas tipo supra-aórticas associadas com obstrução do óstio coronário esquerdo; em parece que a cirurgia direta sobre as lesōes do óstio é uma proposta lógica; entretanto, esta cirurgia, acredito, na maioria dos casos em que nós temos obstrução de óstios, que é na retroca de valvas aórticas, é uma condição na qual não se poderia fazer este tipo de cirurgia e sim a cirurgia de ponte de safena ou mamária. Eu vejo algumas vantagens, como já enumerou o Dr. Luiz Carlos, neste tipo de cirurgia; a primeira seria (eu gostaria de um esclarecimento maior a respeito, por ele) a cardioplegia; ele refere, no trabalho, que fez a cardioplegia, mas gostaria de saber como a fez; tenho a impressão de que, após a abertura do óstio, fez uma cardioplegia seletiva no tronco da coronária esquerda; o outro ponto que eu gostaria de esclarecimento é a respeito de qual o enxerto mais indicado, nessa situação; é mais fácil trabalhar com veia, como ele fez em 3 pacientes, ou então com pericárdio autógeno ou pericárdio heterólogo conservado. Quero parabenizar o grupo do $\mathrm{Dr}$. Luiz Carlos, pelo briIhantismo da exposição, pela documentação desses pacientes e acredito que, na realidade, nesses pacientes com obstrução isolada do óstio, é um método que podemos, perfeitamente, realizar. Muito Obrigado.

\section{DR. ALEXANDRE BRICK Juiz de Fora, $M G$}

É, para nós, uma grande honra e, ao mesmo tempo, uma enorme responsabilidade comentar este trabalho do Dr. Luiz Carlos Bento de Souza, com quem tivemos a oportuniade de conviver de maneira indireta, uma vez que não trabalhamos em seu Serviço, mas que nos deixou até hoje, cada vez mais, novos ensinamentos e, agora, essa responsabilidade aumenta ainda mais, pois estamos frente ao presidente de nossa sociedade. Em virtude desse fato, gostaríamos de prestar a ele a nossa homenagem e, também, do Instituto do Coração de Juiz de Fora. Lembrando palavras de um grande homem, que viveu e morreu sempre procurando servir aos outros, Martin Luther King, que afirmou: "aqueles que lutam em favor dos seus semelhantes um dia construirão aquilo que os egoistas procuram destruir, de qualquer maneira"; e nós temos certeza, Luiz, de que este é, também, o seu pensamento. Por isso, os nossos sinceros cumprimentos. Quanto ao trabalho apresentado, vamos teceralguns comentários e fazer algumas perguntas que já foram feitas aqui pelo Dr. Figueiroa. Realmente, a literatura apresenta poucos casos relatados de lesão isolada do óstio da coronária; (slide) este trabalho já foi referido pelo Dr. Luiz Carlos e pelo Dr. Figueiroa, é a lesão do óstio da coronária direita; aqui está a fotografia da lesão do óstio deste trabalho e aqui está representada a cirurgia que foi apresentada com a plastia de veia safena com este óstio da coronária; em nosso Serviço, em Juiz de Fora, num total de 6.400 cateterismos realizados pelo Dr. João Batista Lopes Soares, foram encontradas 8 destas lesões, com percentual de $0,12 \%$, em concordância com o que foi mostrado pelo Dr. Luiz Carlos e, na revisāo da literatura nós não temos nenhum caso tratado com esta técnica, mas achamos que a opção é ótima, evitando, principalmente, o problema relacionado com as pontes de safena na sua evolução a longo prazo. Em nosso meio, tivemos a oportunidade de levantar 4 casos que foram tratados com esta técnica; é a lesão isolada do óstio da coronária e que foram operadas com técnica semelhante à aqui descrita pelo Dr. Luiz Carlos; 2 pacientes foram operados no Serviço do Dr. Sérgio Almeida de Oliveira e 1 das pacientes, além da lesão do óstio, apresentava lesão da valva mitral, que foi tratada concomitantemente; a outra paciente apresentou, 3 anos após, lesōes nas artérias coronárias, sendo, então, reoperada para revascularizaçāo do miocárdio e, segundo o Dr. Sérgio nos informou, a observação dessa plastia anteriormente realizada estava perfeita; os outros 2 casos nos foram relatados pelo Dr. Ênio Buffolo e, como na experiência do Dr. Sérgio, tratava-se de pacientes jovens, com lesão também isolada do óstio - da coronária esquerda; nesses 2 casos, a técnica empregada pelo Dr. Énio foi um pouco diferente da usada pelo Dr. Luiz Carlos e pelo Dr. Sérgio, pois ele fez a transecção do tronco pulmonar para realizar a plastia, também com veia safena; em todos esses 4 casos que tivemos a oportunidade de levantar através de comunicação verbal, a plastia foi realizada com veia safena. Algumas coisas nós gostaríamos que o Dr. Luiz Carlos comentasse, se isso estaria associado com uma atenção maior do pessoal da hemodinâmica e da cardiologia e do próprio cirurgião pela doença, ou se foi, realmente, uma coincidência; num dos casos aqui apresentados o Dr. Luiz Carlos já comentou isto; foi observada, além da lesão do óstio, a lesão na artéria descendente anterior; perguntamos ao Dr. Luiz Carlos se não seria interessante, nesses casos, realizar a revascularização da arté- 
SOUZA, L. C. B.; CHACCUR, P.; DINKHUYSEN, J. J.; ANGRISANI NETO, S.; ARNONI, A. S.; ABDULMASSIH NETO, C.; BARROSO. H. B.; PAVANELlO, R.; PIEGAS, L. S.; PAULISTA, P. P.; SOUSA, J. E. M. R.; JATENE, A. D. - Tratamento cirúrgico da lesão do óstio da coronária esquerda. Rev. Bras. Cir. Cardiovasc., 3(3): 182-188, 1988.

ria; outro ponto que gostaria que fosse explicado e que o Dr. Figueiroa também já perguntou, mas vamos reforçar, é quanto à introdução da cardioplegia, se ela teria sido feita na raiz da aorta, ou diretamente no óstio da coronária, após abordagem e perguntamos também se notou alguma diferença técnica imediata na lesāo entre os pacientes que receberam a veia safena e o pericárdio bovino; finalmente, mostraremos aqui 2 casos de nossa casuística, com lesão do óstio da coronária pós-cirurgia de troca valvar aórtica tratados com a técnica de revascularizaçāo do miocárdio e perguntaríamos ao Dr. Luis Carlos se, nesses casos, indicaria esse tipo de conduta para corrigir a lesão do óstio. Agradecemos a oportunidade dada pela comissāo organizadora e parabenizamos, mais uma vez, o Dr. Luiz Carlos, pelo excelente trabalho. Muito obrigado.

\section{DR. SOUZA}

(Encerrando)

Inicialmente, quero agradecer aos comentadores. Eu responderia ao Carlos e, ao mesmo tempo ao Alexandre, que a realizaçāo deste tipo de operaçāo com uma prótese aórtica em posiçâo torna-se mais desconfortável; não acredito seja impossivel; aceito, com muito mais razão, nesse caso, que se propuzesse uma revascularizaçāo nos moldes habituais do que a cirurgia sobre o óstio; entretanto, não concluiria pela impossibilidade do procedimento, mas reconheço que ela seria mais trabalhosa, pela presença de uma estrutura rígida numa regiāo onde se tenha que afastar e trabalhar; o problema da cardioplegia é que ela foi uma cardioplegia de raiz de aorta, não foi uma cardioplegia ostial após liberação de estenose, nós acreditamos que, por esse óstio, passava sangue em quantidade suficiente para promover uma funçāo miocárdica compatível com a vida e nāo vejo porque, durante a cirurgia, ao se injetar uma solução cardioplégica sobre pressão na raiz da aorta, que essa soluçāo não possa ganhar o leito coronário e promover o resfriamento do miocárdio; no que diz respeito ao enxerto, eu tenho preferência pela veia; creio que um segmento de veia safena elítico traz um bom elemento de ampliação para essa área; eu não procuraria utilizar o pericárdio autógeno porque, algumas vezes em que ele foi utilizado, já se percebeu a capacidade de enrugamento e retração desse pericárdio e eu não sei se, talvez, isso pudesse ocorrer nessa posição, o que seria um desastre, pois que o doente voltaria a ter todos os inconvenientes outra vez e também aceito que o pericárdio tratado seja uma solução para esse enxerto, mas tenho minha preferência pelo segmento de veia. Nāo encontramos, na evolução até agora, nenhuma diferença entre o doente que recebeu pericárdio bovino e os outros com safena como elemento de ampliação; não há nenhuma diferença funcional, desde que nós nāo pudemos ter o elemento anatômico do reestudo, pela recusa dos doentes, pelo menos a avaliação dos doentes não mostrou nenhuma diferença no comportamento desses dois enxertos. Sobre a associação de lesōes a essa lesão ostial, neste caso a opção foi por ataque direto ao tronco óstio, porque, fatalmente, esse doente nāo tinha uma lesão arterial que justificasse qualquer tipo de atitude na artéria e, realmente, era uma lesão discreta; hoje, tenho a certeza de que aquele acidente coronário havido no pós-operatório foi por isquemia em cima de uma placa de $20 \%$ a $30 \%$; creio que, nessas circunstâncias, eu também aceito a discussâo, há controvérsias sobre se seria melhor operar sobre as porções de lesão da artéria, ou se fazer uma cirurgia central; acredito que, em casos como este apresentado, que tinha uma lesão discreta na descendente anterior, eu voltaria a realizar a ampliação da lesão do óstio e somente pensaria em abandonar a ampliação do óstio para um ataque às porções periféricas da artéria, quando a lesão assumisse magnitude maior. Por fim, eu queria agradecer as palavras de entusiasmo à direção da SBCCV, porque, realmente eu estarei, nestes próximos 2 anos, muito ocupado com este assunto. Muito obrigado. 